\title{
A case of locked-in syndrome complicated by central deafness
}

\author{
Colette M Smart* , Joseph T Giacino, Tara Cullen, Diana Rodriguez Moreno, Joy Hirsch, Nicholas D Schiff \\ and Martin Gizzi
}

\section{SUMMARY}

Background A 53-year-old male with a history of hypertension, diabetes mellitus, and factor $\mathrm{V}$ deficiency presented to an emergency room with progressively increasing headache, slurred speech, and left upper extremity weakness. Over the previous 3 months, he had been receiving warfarin for prophylaxis of deep venous thrombosis following knee surgery. After presentation and an initial period of coma, he became tetraplegic and anarthric, requiring intubation and ventilatory assistance.

Investigations Neurological examination, CT scan, electroencephalogram, brainstem auditory and visual evoked potential studies, neuropsychological assessment and functional MRI studies.

Diagnosis Locked-in syndrome following ventral pontine hemorrhage, complicated by central deafness secondary to extension of the lesion to the inferior colliculus.

Management Development of an augmentative communication system designed to exploit the patient's preserved cognitive and motor functions.

KEYWORDS central deafness, disorders of consciousness, locked-in syndrome, neuropsychological testing, stroke

OME

CM Smart is a Postdoctoral Fellow in Clinical Neuropsychology at the JFK Johnson Rehabilitation Institute and the New Jersey Neuroscience Institute, JFK Medical Center, Edison, NJ, JT Giacino is an Associate Professor in the Department of Neuroscience at Seton Hall University, South Orange, NJ, and Associate Director of Neuropsychology at JFK Johnson Rehabilitation Institute and the New Jersey Neuroscience Institute, JFK Medical Center, Edison, NJ, TCullen is a Speech-Language Pathologist at the JFK Johnson Rehabilitation Institute, Edison, NJ, D Rodriguez Moreno is a Postdoctoral Fellow in the fMRI Research Center at Columbia University, New York City, NY, J Hirsch is a Professor in the Departments of Radiology, Neuroscience and Psychology, and Director of the fMRI Research Center at Columbia University, New York City, NY, ND Schiff is Associate Professor of Neurology and Neuroscience in the Department of Neurology and Neuroscience at Weill Cornell Medical College, New York, NY, and M Gizzi is Professor and Chairman of Neuroscience, Seton Hall University School of Graduate Medical Education, South Orange, NJ, and Chairman of the New Jersey Neuroscience Institute, JFK Medical Center, Edison, NJ, USA.

\section{Correspondence}

*New Jersey Neuroscience Institute, JFK Medical Center, 65 James Street, Edison, NJ 08818, USA csmart@solarishs.org

Received 30 November 2007 Accepted 16 April 2008 Published online 27 May 2008

www.nature.com/clinicalpractice

doi:10.1038/ncpneuro0823

\begin{abstract}
Medscape Continuing Medical Education online Medscape, LLC is pleased to provide online continuing medical education (CME) for this journal article, allowing clinicians the opportunity to earn CME credit. Medscape, LLC is accredited by the Accreditation Council for Continuing Medical Education (ACCME) to provide CME for physicians. Medscape, LLC designates this educational activity for a maximum of 0.5 AMA PRA Category 1 Credits $^{T M}$. Physicians should only claim credit commensurate with the extent of their participation in the activity. All other clinicians completing this activity will be issued a certificate of participation. To receive credit, please go to http://www.medscape.com/cme/ncp and complete the post-test.
\end{abstract}

\section{Learning objectives}

Upon completion of this activity, participants should be able to:

1 Identify the most common cause of locked-in syndrome.

2 Describe the diagnostic assessment of suspected locked-in syndrome.

3 List clinical elements of classic locked-in syndrome.

4 Identify effective therapies for patients with locked-in syndrome.

\section{Competing interests}

The authors and the Journal Editor $\mathrm{H}$ Wood declared no competing interests. The CME questions author CP Vega declared that he has served as an advisor or consultant to Novartis, Inc.

\section{THE CASE}

A 53-year-old right-handed male with a history of hypertension, diabetes mellitus, hyperlipidemia, and factor $\mathrm{V}$ deficiency presented to an emergency room with headache, slurred speech, and left upper extremity weakness and numbness, all of which had progressively increased in severity over a period of several hours. At the time of presentation, the patient was receiving warfarin ( $4 \mathrm{mg} /$ day) for prophylaxis of deep venous thrombosis following knee surgery 3 months previously. His initial Glasgow Coma Scale score was 12, but his level of consciousness declined sharply in the emergency room (Glasgow Coma Scale score $=4$ ), ultimately resulting in the need for ventilator insertion. On admission to an intensive care unit the following morning, the patient was comatose, with no spontaneous eye opening or response 
to command, and without oculocephalic and corneal reflexes. His spontaneous eye movements were limited to intermittent periods of ocular bobbing. Twenty-four hours later, the patient recovered spontaneous eye opening but still had no response to command and demonstrated decerebrate posturing to noxious stimuli. An initial head CT scan in the emergency department revealed a ventral midline hemorrhage extending from the pontomedullary junction to the upper pons (hemorrhaging was greater on the right than on the left). It was presumed that the patient had suffered a hemorrhagic stroke secondary to use of warfarin for deep venous thrombosis prophylaxis. At $36 \mathrm{~h}$, there was further rostral extension of the hemorrhage to the inferior midbrain and expansion into the left pons and midbrain. A repeat CT scan on day 3 (Figure 1) revealed an additional small area of hemorrhage that involved the dorsal pontomesencephalic region, including the inferior colliculi (particularly on the right), in addition to tegmental edema.

By day 7, vertical pursuit eye movements and eye movements in response to command were noted, although these behaviors were difficult to discern because of near-constant ocular bobbing. The patient remained tetraplegic and anarthric. Assessment of the patient's voluntary eye movement on day 10 by use of the JFK Coma Recovery Scale-Revised ${ }^{1}$ confirmed intact vertical pursuit and reproducible eye movement to command. These behaviors were consistent with the diagnostic criteria for a minimally conscious state (MCS), ${ }^{2}$ although the results from electroencephalogram, brainstem auditory evoked response (BAER) and visual evoked response studies, conducted approximately 5 weeks following the stroke, were normal, which is unusual during an MCS. In view of the large pontine lesion, a diagnosis of locked-in syndrome (LIS) was considered.

At approximately 4 months following the stroke, improved arousal and recovery of lateral head movements enabled further investigation of the patient's cognitive status. Over a period of 2 weeks, in up to 3 sessions per day, the patient was exposed to simple situational orientation questions, accompanied by visual cues. The patient's yes or no responses were obtained by use of shouldermounted toggle switches that were activated by lateral head movements. Overall response consistency and accuracy during this assessment period were $99 \%$ and $98 \%$, respectively (Box 1).

At approximately 6 months after the stroke, verbally presented questions that required access
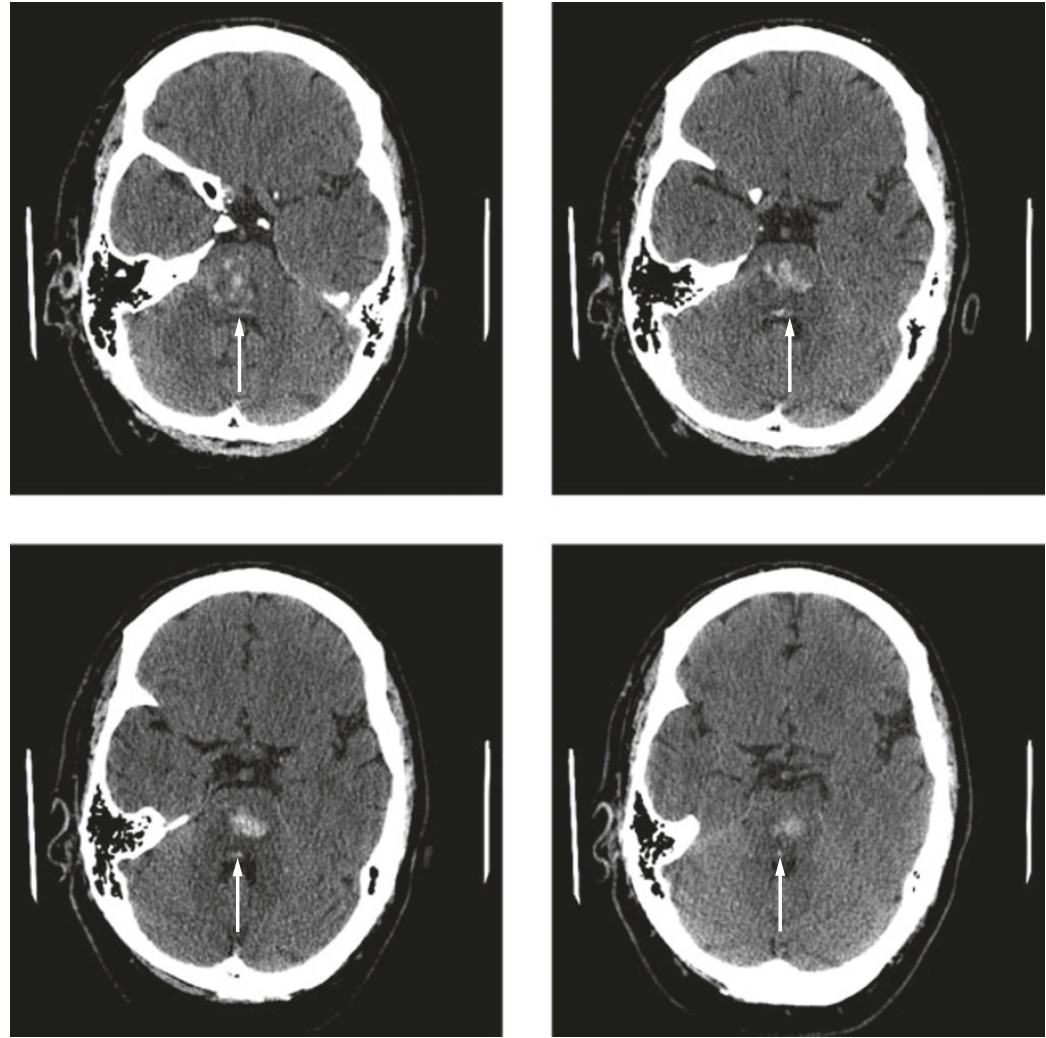

Figure 1 CT scans of the brain at 3 days following stroke in a patient with locked-in syndrome. Four axial scans show a hemorrhagic infarct of the brainstem interrupting the auditory processing pathway at the level of the inferior colliculi (arrows).

Box 1 Neurobehavioral assessment of a patient with locked-in syndrome at 4 months following onset of the condition.

- Question type: situational orientation (verbal and visual)

- Sample questions: "Am I clapping my hands right now?"; "Am I touching my nose right now?"

- Total number of trials: 29 sessions $\times 12$ trials $=348$ trials

- Across-trial consistency: 344 out of 348 ( 4 failures to respond) $=99 \%$

- Accuracy of responses: 336 out of 344 (8 incorrect responses) $=98 \%$

to semantic knowledge were added to the cognitive assessment protocol. The patient's accuracy and consistency of responses to simple situational orientation questions remained high, but response accuracy was unexpectedly poor on verbally presented semantic knowledge questions (Box 2). 
Box 2 Neurobehavioral assessment of a patient with locked-in syndrome at 6 months following onset of the condition.

- Question types: situational orientation (verbal and visual); verbally presented semantic knowledge

- Sample questions: "Is a rose a flower?"; "Is ice hot?"

- Total number of trials: 20 sessions $\times 18$ trials $=360$ trials

- Across-trial consistency: 358 out of 360 ( 2 failures to respond) $=99 \%$

- Accuracy of verbal situational question responses: 117 out of 119 ( 2 incorrect responses) $=98 \%$

- Accuracy of visual situational question responses: 112 out of 120 (8 incorrect responses) $=93 \%$

- Accuracy of verbal semantic question responses: 65 out of 119 (54 incorrect responses) $=55 \%$

To clarify the reason for the patient's poor performance on semantic knowledge questions, visually based semantic knowledge questions were added to the cognitive assessment protocol (e.g. the examiner showed a picture of ice cream and asked, "Is this hot?") at 7 months following the stroke. The patient's performance accuracy was $92 \%$ on visually based semantic knowledge items as compared with $67 \%$ on verbally based questions.

Contemporaneous to the assessment of the patient's semantic knowledge, at around 6 months after the stroke, the patient began using a spelling board. By use of this communication device, he reported that his sense of hearing seemed distorted (e.g. "all is loud") and that speech sounded muffled, although he had no premorbid hearing deficit. He also reported being able to understand verbal information much better when provided in written form. Neurobehavioral assessment was, therefore, repeated at 7 months after the stroke, with verbal semantic items presented with or without a written transcript. The findings revealed high accuracy on verbal semantic items presented both with and without a written transcript (100\% and 95\%, respectively). However, the patient's response accuracy to yes or no questions was poor without the aid of written transcripts during interactions conducted outside the formal assessment protocol. It was unclear whether the general inconsistency between the patient's response to verbal versus visual questioning was attributable to cognitive impairment or a disturbance in auditory processing.

At approximately 10 months following the stroke, the patient underwent neuropsychological testing based on a protocol proposed by Neumann and Kotchoubey for cognitive assessment of severely paralyzed patients. ${ }^{3}$ Specifically, the patient's general fund of knowledge, receptive language, memory, and executive functioning were examined. All test instructions were presented in visual form, and the patient used a spelling board during tests that required open-ended responses. Premorbid intellectual function was estimated to be in the average range. On tests of discrete cognitive functions, the patient's performance was within normal limits on all measures except rate of new learning (Table 1), indicating that higher-level cognitive functions were essentially intact.

At approximately 1 year following the stroke, functional MRI (fMRI) studies were performed to assess the integrity of the patient's neural networks that underlie auditory and visual processing. ${ }^{4-6}$ In a hierarchical scanning paradigm, the patient was exposed to passive auditory stimulation under two conditions (intelligible speech and unintelligible, reversed speech) followed by passive visual stimulation under two conditions (landscapes and faces). Instructions for all tasks were presented in written form. Standard blood oxygen leveldependent (BOLD) signal analyses were performed by use of the statistical parametric mapping package SPM2 (Wellcome Department of Imaging Neuroscience, Institute of Neurology, University College London, London, UK).

The passive speech paradigm failed to elicit the expected activation of the superior temporal gyrus (the primary structure responsible for mediating language comprehension) during the normal speech condition (Figure 2A). Moreover, activation of the primary auditory cortex, which is responsible for basic sound reception, was weak and inconsistent across both language conditions, suggesting impaired discrimination of speech from background scanner noise. Findings from the passive visual paradigm revealed activation of the primary visual cortex with selective activation of the fusiform gyrus and parahippocampal place areas during the face and landscape conditions, respectively (Figure 2B). These results were indicative of well-preserved visual processing pathways. After completion of the fMRI studies, the patient's medical status deteriorated, preventing further assessment. 


\section{DISCUSSION OF DIAGNOSIS}

The weight of the evidence obtained from the patient-presentation with anarthria and tetraplegia in the context of a brainstem stroke; normal performance on neuropsychological measures administered nonverbally; and failure to activate auditory cortices, coupled with normal activation of visual association areas, on fMRI-was suggestive of LIS complicated by central deafness.

The term "locked-in syndrome" was initially coined by Plum and Posner in 1966, ${ }^{7}$ although the condition has been variously referred to as cerebromedullospinal disconnection, de-efferented state, pseudocoma, and ventral pontine syndrome. The most frequently reported premonitory symptoms of LIS include headache, dizziness, vertigo, and hemiparesis. ${ }^{7}$ LIS most commonly occurs after lesions of the ventral pons, although other etiologies such as trauma, tumor, and encephalitis have been documented (Box 3). ${ }^{8}$ The cardinal signs of LIS include tetraplegia, lower cranial nerve palsy, and anarthria, with preserved consciousness.

Accurate diagnosis of LIS in the early stages is critical for establishing a prognosis and appropriate management strategies. However, patients with LIS might initially be comatose, which can delay the diagnosis. ${ }^{9}$ Injury to tegmental regions of the upper pons and midbrain increases the likelihood of initial disturbances in consciousness, but CT scans completed in the acute stage of injury are limited in their capacity to detect edema or regional infarction in these brain structures. In the present patient, the lesions evident in the inferior colliculi suggested that there was an interruption of the tegmental reticular system, which might account for the arousal fluctuations noted in the acute post-coma phase.

Following emergence from coma, most patients transition to a vegetative state or an MCS before normal consciousness is regained. Patients with LIS might be misdiagnosed as being in a vegetative state because of the attendant anarthria and tetraplegia. It might also be difficult to differentiate LIS from an MCS when the patient exhibits fluctuating arousal and oculomotor dysfunction, which can interfere with vertical eye-movement control, a feature that is often the best early sign of preserved consciousness in LIS. Assessment can be further confounded by the fact that patients with LIS do not retain sufficient strength or motor control to meet existing criteria for
Table 1 Results of neuropsychological assessment in a patient with locked-in syndrome.

\begin{tabular}{|c|c|c|}
\hline Neuropsychological domain & Raw score & Interpretation \\
\hline \multicolumn{3}{|l|}{ Premorbid function } \\
\hline Barona IQ & 97.87 & Average \\
\hline \multicolumn{3}{|l|}{ Receptive language } \\
\hline Complex Ideation from the BDAE & 11 out of 12 & $T=46$ (normal) \\
\hline \multicolumn{3}{|l|}{ Current intellectual function } \\
\hline Information from the WAIS-R NI & 24 out of 29 & $\mathrm{SS}=13($ normal $)$ \\
\hline \multicolumn{3}{|l|}{ Memory } \\
\hline \multicolumn{3}{|l|}{ CVLT-II SF } \\
\hline Trials $1-4$ total & 15 & $T=24$ (impaired) \\
\hline Short delay free recall & 4 & $Z=-2$ (impaired) \\
\hline Long delay free recall & 6 & $Z=0$ (normal) \\
\hline Hits & 9 & $Z=0.5$ (normal) \\
\hline False positives & 1 & $Z=0.5$ (normal) \\
\hline Recognition discriminability & 3.2 & $Z=0.5$ (normal) \\
\hline \multicolumn{3}{|l|}{ Executive function } \\
\hline \multicolumn{3}{|l|}{ WCST-64 } \\
\hline Categories completed & 1 & $6-10^{\text {th }}$ percentile (normal) \\
\hline Trials to first & 27 & $11-16^{\text {th }}$ percentile (normal) \\
\hline Total errors & 27 & $10^{\text {th }}$ percentile (normal) \\
\hline Perseverative responses & 16 & $21^{\text {st }}$ percentile (normal) \\
\hline Perseverative errors & 15 & $16^{\text {th }}$ percentile (normal) \\
\hline Nonperseverative errors & 12 & $10^{\text {th }}$ percentile (normal) \\
\hline Conceptual level responses & 30 & $7^{\text {th }}$ percentile (normal) \\
\hline
\end{tabular}

Abbreviations: BDAE, Boston Diagnostic Aphasia Examination; CVLT-II SF, California Verbal Learning Test Second Edition, Short Form; SS, scaled score; T, T-score; WAIS-R NI, Wechsler Adult Intelligence Scale-Revised as a Neuropsychological Instrument; WCST-64, Wisconsin Card Sorting Test-64 card version; Z, Z-score.

emergence from an MCS (i.e. demonstration of functional communication and object use).

Bauer and colleagues have categorized LIS as classical, incomplete, or total (Box 4). ${ }^{10}$ According to this classification, the present patient emerged from a coma into classical LIS. The only behavioral evidence of conscious awareness he retained was the ability to execute vertical eye movements to command. However, this behavior also technically satisfies the diagnostic criteria established for an MCS. Although it was possible to elicit reproducible eye movements to command, the patient's fluctuations in arousal and persistent ocular bobbing notably compromised the consistency of the responses. It was not until the arousal disorder and ocular 

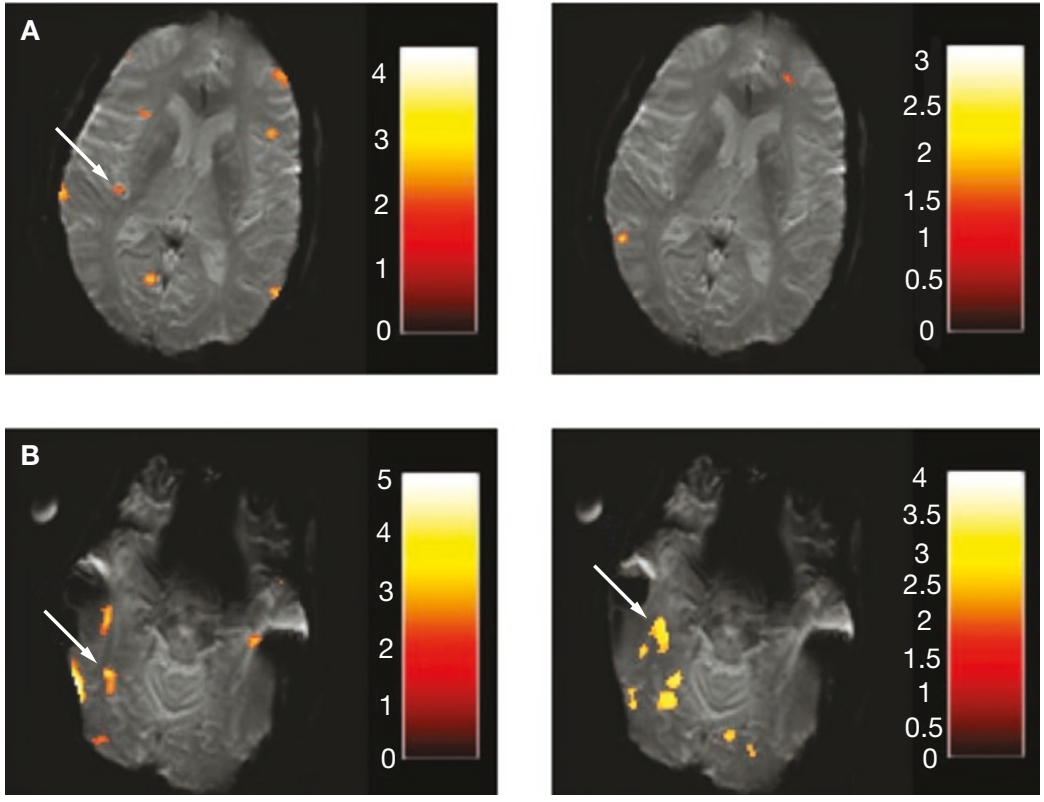

Figure 2 Functional MRI images of a patient with locked-in syndrome. The images were acquired according to standard clinical procedures for passive stimulation techniques. ${ }^{5,6}$ Instructions to the patient were given before each run in the form of written text. (A) Colored voxels indicate BOLD responses elicited by exposure to an auditory narrative (normal forward speech, left panel), and the same narrative presented as filtered sound (reversed speech, right panel). The primary auditory cortex shows a weak unilateral signal (arrow) in response to the forward speech. There is no clear evidence of activity in the left hemisphere superior temporal gyrus (putative Wernicke's area) in any of the conditions. (B) Colored voxels indicate BOLD responses elicited by exposure to visually presented black and white pictures of faces (left panel) and black and white pictures of landscapes (right panel). The activities in the right inferior temporal gyrus (face fusiform area, left panel, arrow) and the right parahippocampal gyrus (parahippocampal place area, right panel, arrow) are consistent with preserved specificity of the visual system. Color bars reflect the range of BOLD signal intensity changes with corresponding $T$-values. Abbreviation: BOLD, blood oxygen level-dependent.

Box 3 Common etiologies of locked-in syndrome. ${ }^{8}$
Vascular etiologies
- $\quad$ Basis pontis infarct
- $\quad$ Pontine hemorrhage
- $\quad$ Transient ischemia
- Basilar artery occlusion
- Hypoxic-ischemic events
Nonvascular etiologies
- Trauma
- Central pontine myelinolysis
- Tumor
- Encephalitis
- Toxins
- Multiple sclerosis affecting the ventral pons

bobbing resolved that he was able to consistently follow eye-movement commands. Over time, the patient's condition was deemed to have evolved into incomplete LIS on the basis of his recovery of lateral head movements, but it was not possible to reliably determine cognitive status until 10 months after stroke onset.

The assessment of hearing is of critical importance in LIS, as speech and gestural modes of communication are either severely compromised or unavailable. Unfortunately, there is a paucity of literature available on auditory processing in individuals with LIS. Aside from Jean-Dominique Bauby's autobiographical account of distorted hearing after a brainstem stroke, ${ }^{11}$ we could find only one reported case documenting "deafness" subsequent to LIS. ${ }^{12}$ In that case, the patient showed no response to pure tones of any frequency or loudness and no response to speech. ${ }^{12}$

In the present case, the patient's difficulty in comprehending spoken language was probably attributable to central deafness, as indicated by the normal results from BAER studies (an observation that has been reported previously in patients with central deafness arising from brainstem lesions involving the inferior colliculi $\left.{ }^{13,14}\right)$, the patient's yes or no responses to questioning, neuropsychological test results, and the lack of activation in primary auditory cortices as observed in the fMRI studies. The structural and functional neuroimaging findings in this patient suggest that the mechanism underlying the central deafness is best explained by a disconnection syndrome in which the inferior collicular lesion interrupted the auditory pathway from the mesodiencephalic region to the primary auditory cortices.

Access to paralinguistic aspects of speech in the form of visual cues might explain why the patient did so well on initial situational orientation items (all of which were accompanied by a visual stimulus) and why his response accuracy to verbal semantic questions improved over a 3-month period (this improvement might have been mitigated by lip-reading practice effects ${ }^{15}$ ).

In view of the high margin of error for early misdiagnosis of LIS, serial neurological examinations combined with neuropsychological assessments adapted for use in nonverbal patients with quadriparesis are essential to accurately ascertain the level of consciousness and cognition. In the present patient, underarousal, oculomotor disturbance, and 
Box 4 Classification of locked-in syndrome. ${ }^{10}$

Classical

- Follows Plum and Posner's ${ }^{7}$ original description of LIS, characterized by anarthria, tetraplegia, and lower cranial nerve palsy, with voluntary vertical eye movements and preserved consciousness

Incomplete

- Includes all the features of classical LIS, with some additional motor recovery (e.g. distal limb movement, horizontal eye movements)

Total

- Similar to classical LIS, but without voluntary vertical eye movements

Abbreviation: LIS, locked-in syndrome.

impaired auditory processing conspired to confound diagnostic assessment. Thus, serially conducted assessments of possible diagnostic confounders are necessary to accurately characterize cognitive function in LIS.

\section{TREATMENT AND MANAGEMENT}

Although there are instances in which LIS is reversible, there is no treatment known to alter the pace of recovery or final outcome for individuals with this condition. Regardless, establishment of a functional communication system should be a priority for all patients with LIS. The present patient relied on a spelling board, but a range of other augmentative communication devices is also available. These include eye-gaze computer systems ${ }^{9}$ and speech-generating devices that can be utilized by patients with residual motor recovery. The present patient learned to activate shoulder-mounted toggle switches connected to an assistive communication device, enabling him to independently initiate discourse and communicate his needs and feelings. Unfortunately, as illustrated here, undetected auditory processing impairment can confound the assessment of cognition and the capacity for functional communication. Simple bedside screening procedures can be employed to mitigate this problem, including comparing a patient's response consistency and accuracy to verbal questions presented within versus outside the patient's field of vision. If impaired auditory processing is suspected, audiometry studies should be conducted and appropriate accommodations prescribed, such as provision of information in a written format to facilitate communication.

\section{CONCLUSIONS}

LIS, which is typically caused by lesions in the ventral pons, manifests as anarthria and quadriplegia with preserved vertical eye movements. Patients with LIS retain consciousness and, with intensive early management, can survive for many years. This report highlights the importance of conducting serial neurological examinations and of adapting neuropsychological assessment measures for use in nonverbal patients with quadriparesis, and the article illustrates the emerging role of neuroimaging in differential diagnosis and in the investigation of higher-level cognitive functions. Such an approach to assessment might help to avoid underestimation of a patient's cognitive capacity, a situation which can have a negative impact on the patient's quality of life and lead to inappropriate treatment decisions. ${ }^{9}$

\section{References}

1 Giacino JT et al. (2004) The JFK Coma Recovery Scale-Revised: measurement characteristics and diagnostic utility. Arch Phys Med Rehabil 8: 2020-2029

2 Giacino JT et al. (2002) The minimally conscious state: definition and diagnostic criteria. Neurology 58: 349-353

3 Neumann N and Kotchoubey B (2004) Assessment of cognitive functions in severely paralysed and severely brain-damaged patients: neuropsychological and electrophysiological methods. Brain Res Brain Res Protoc 14: 25-36

4 Hirsch J et al. (2001) Interconnected large-scale systems for three fundamental cognitive tasks revealed by functional MRI. J Cogn Neurosci 13: 389-405

5 Schiff ND et al. (2005) fMRI reveals large-scale network activation in minimally conscious patients. Neurology 64: $514-523$

6 Giacino JT et al. (2006) Functional neuroimaging applications for assessment and rehabilitation planning in patients with disorders of consciousness. Arch Phys Med Rehabil 87 (Suppl 1): S67-S76

7 Plum F and Posner JB (1966) The Diagnosis of Stupor and Coma. Philadelphia: FA Davis Company

8 Patterson JF and Grabois M (1986) Locked-in syndrome: a review of 139 cases. Stroke 17: 758-764

9 Laureys S et al. (2005) The locked-in syndrome: what is it like to be conscious but paralyzed and voiceless? Prog Brain Res 150: 495-511

10 Bauer $\mathrm{G}$ et al. (1979) Varieties of the locked-in syndrome. J Neurol 221: 77-91

11 Bauby JD (1998) The Diving Bell and the Butterfly: A Memoir of Life in Death. New York: Vintage International

12 Keane JR (1985) Locked-in syndrome with deafness. Neurology 35: 1395

$13 \mathrm{Hu}$ C et al. (1997) Traumatic brainstem deafness with normal brainstem auditory evoked potentials. Neurology 48: 1448-1451

14 Vitte E et al. (2002) Midbrain deafness with norma brainstem auditory evoked potentials. Neurology 58: 970-972

15 Riley JA and Cogan GB (2007) A two-mechanism model of pure word deafness. In University of Maryland Working Papers in Linguistics, edn 16, 201-221 (Eds Omaki A et al.) College Park: UMWPiL
Acknowledgments

JT Giacino's contribution to this work was supported in part by the National Institute on Disability and Rehabilitation Research (award \#H133A070030). We thank the patient's treating physician for facilitating the patient's participation in the study and assisting with coordination of study-related activities. We are also indebted to the rehabilitation team for assisting with development and implementation of the augmentative communication system and, most of all, to the patient's family members for their untiring dedication and support. Charles P Vega, University of California, Irvine, CA, is the author of and is solely responsible for the content of the learning objectives, questions and answers of the Medscapeaccredited continuing medical education activity associated with this article.

Competing interests The authors declared no competing interests. 